\title{
Identity, Indiscernibility, and Philosophical Claims
}

\author{
Décio Krause* Antonio M. N. Coelho \\ Grupo de Lógica e Fundamentos da Ciência \\ Departamento de Filosofia \\ Universidade Federal de Santa Catarina
}

\begin{abstract}
The concept of indiscernibility in a structure is analysed with the aim of emphasizing that in asserting that two objects are indiscernible, it is useful to consider these objects as members of (the domain of) a structure. A case for this usefulness is presented by examining the consequences of this view to the philosophical discussion on identity and indiscernibility in quantum theory.
\end{abstract}

Keywords: identity, indiscernibility, invariance, quantum objects, structures.

\section{Introduction}

The significance of Leibniz's Principle of the Identity of Indiscernibles (PII) in quantum mechanics has been widely discussed in the philosophical literature, mainly in connection with the problem of whether or not it is violated in the quantum domain. Elementary 'identical' quantum objects in the same state of motion would be examples of entities which differ solo numero, having all their intrinsic properties in common and being so that in certain situations even spatio-temporal location cannot be used to distinguish among them (for instance when they are in 'entangled states'). This would constitute a motive for PII to be false. ${ }^{1}$

A version of Leibniz's principle can be written in a second order language as

$$
\forall F(F(a) \leftrightarrow F(b)) \rightarrow a=b
$$

where $a$ and $b$ denote individuals and $F$ is a variable ranging over properties of individuals. The converse of this principle is known as the Principle of the Indiscernibility of Identicals (II), namely,

\footnotetext{
* Partially supported by CNPq.

${ }^{1}$ See French (1989) for a detailed discussion.
} 


$$
a=b \rightarrow \forall F(F(a) \leftrightarrow F(b))
$$

and their conjunction gives Leibniz Law, which is in general taken as meaning that identity (being the same object, in symbols, ' $a=b$ ') is defined by means of indiscernibility (agreement with respect to all properties). ${ }^{2}$

In the quantum realm the discussion concerning the domain of the variable $F$ acquires a particular importance, for it raises the problem of what should be considered as a legitimate property of a quantum object, and in considering some of the possibilities, at least three forms of PII have been distinguished elsewhere: PII(1), the weakest form, states that it is not possible for two individuals to have all properties and relations in common; PII(2) excludes the spatio-temporal properties, while PII(3) includes only monadic, non-relational properties, and much dispute has been presented on the predominance of one of these forms of PII over the others; see French 1989 and French 1999 for more details.

In this paper we shall consider aspects of this discussion from a perspective which does not have been much considered in the philosophical literature, but which we guess may be of importance for a full understanding of the involved problems, in particular, those related to the philosophical discussions regarding quantum physics and concepts like identity, indiscernibility, and individuality. Our approach could be said to be 'model theoretic' in a certain sense, since we shall be concerned mainly with the mathematical structures involved in such a discussion. In short, we shall push the context to mainly its logical and mathematical consequences and implicit assumptions, with the hope that it can be useful in philosophical issues concerning the foundations of quantum theory. The recalling of some of the basic definitions and ways of approaching identitylike concepts below has the only aim of keeping the paper more self-contained and fixing some terminology.

\section{Characterizing identity}

The standard way of considering the concept of identity in mathematics is linked to a tradition which goes back at least to Leibniz. Frege took possession of Leibniz's dictum Eadem sunt quorum unum potest substitui alteri salva veriate to motivate his 'definition' of identity (equality) in Die Grundlagen der Arithmetik (Frege 1884, §65) but, as remarked by A. Church, he made a confusion between use and mention, for what is to be substituted are not the things themselves, but their names: "things are identical if the name of one can be substituted for that of the other without loss of truth" (Church 1956, p. 300).

As Church also notes, the confusion between use and mention was corrected nine years later in the Vol. 1 of Frege's Grundgesetze der Arithmetik (Frege 1893) but, there, instead of a definition, what we find is an axiom, written by

\footnotetext{
${ }^{2}$ There is no general agreement concerning the terminology used here, for some authors prefer to call 'Leibniz Law' the expression (F2) below.
} 
Church as $\varphi(x=y) \supset \varphi((F)[F(x) \supset F(y)]) .{ }^{3}$ Previously in the Begriffsschrift (Frege 1879), Frege used axioms which can be written as (for any $a$ and $b$ ):

(F1) $a=a$

$(\mathrm{F} 2) a=b \rightarrow(F(a) \rightarrow F(b))$

It should be realized that, by that time, there was not a clear distinction between first and higher order languages, and today we know that Frege's foundational system encompasses 'more' than first order mathematics. The axiom mentioned by Church resembles Whitehead and Russell's definition of identity in Principia Mathematica, although these authors restricted their definition to the so-called 'predicative properties' (predicative propositional functions), so that the application of identity in their system depends on the axiom of reducibility (Whitehead \& Russell 1927, p. 57).

The axioms (F1) and (F2) above are essentially those used in first order languages when identity is taken as an element of the underlying logic but, for expressing the general case (involving formulas in general), $F(a)$ must be read as a formula whatever, while $F(b)$ comes from $F(a)$ from the substitution of some free occurrences of $a$ by $b$, provided that $b$ is free for $a$ in $F(a)$, where $a$ and $b$ are terms whatever; see (Mendelson 1997, p. 95).

If a first order language has only a finite number of predicates, then identity can be defined. For doing that it suffices to write as a formula $A(x, y)$ the conjunction of all possible substitutions in the predicates, in a sense that there is an exhaustion of all the primitive predicates of the theory (Hilbert and Ackermann 1950, pp. 107ff). Then identity between $x$ and $y(x=y)$ is defined by such a formula. For instance, suppose that the only primitive predicates are the binary predicate $P$ and the unary predicate $Q$. Then $A(x, y)$ should be the following formula (except by the quantifiers)

$$
(P(x, z) \leftrightarrow P(y, z)) \wedge(P(z, x) \leftrightarrow P(z, y)) \wedge(Q(x) \leftrightarrow Q(y))
$$

which 'simulates' identity in the sense that $x$ and $y$ share all the primitive predicates of the language. A relevant case is that of set theory, where identity $(x=y)$ can be defined by (Fraenkel et al., p. 27):

$$
\forall z(z \in x \leftrightarrow z \in y) \wedge \forall z(x \in z \leftrightarrow y \in z) .
$$

The 'first order' axioms F1 and F2 above have interesting consequences, mainly if we consider semantics. Firstly, let us recall that F1 expresses the intuitive idea that 'every object is identical to itself', and is known as the Reflexive Law of Identity, while F2 is the Substitutivity Law. If we intuitively think of identity as referring to something an object has to itself and to nothing more (the consideration of whether this 'something' is a legitimate 'property' or not

\footnotetext{
${ }^{3}$ See Church 1956, p. 300, fn. 502. Frege's original notation was based in his Begriffsschrift (Frege 1879), and will not be used here.
} 
shall be mentioned below), then we could expect that the semantical interpretation of the identity predicate ' $=$ ' should be the diagonal of the domain of discourse, namely, the set

$$
\Delta_{D}=\{\langle x, x\rangle: x \in D\}
$$

where $D$ stands for the domain of the interpretation.

But F1 and F2 do not 'characterize' the diagonal without ambiguity. Since this result is important and it is not usually mentioned in the philosophical discussions on the subject, let us see in brief some aspects of such a claim. Suppose that $\mathcal{A}=\langle D, \rho\rangle$ is a model for our first order language $L$ in the standard sense, where $\rho$ is the denotation function defined as usual, that is, such that, for every individual constant $c$ of the language, $\rho(c)$, which we denote by $c^{D}$, is an element of $D$; furthermore, for every $n$-ary predicate $R, \rho(R)=R^{D}$ is a subset of the set $D^{n}$; and for any $n$-ary functional symbol $f, \rho(f)$ is a mapping from $D^{n}$ to $D$.

The relation which interprets the primitive symbol of identity is in particular an equivalence relation (since it must be reflexive by F1 and symmetric and transitive, as it can be proven without difficulty (Mendelson loc. cit.)). Let us denote by $\approx_{D}$ such a relation. If $\mathcal{A}^{\prime}=\left\langle D^{\prime}, \rho^{\prime}\right\rangle$ is another interpretation for our language such that its domain $D^{\prime}$ is the quotient set of $D$ by the relation $\approx_{D}$ (that is, $\left.D^{\prime}=D / \approx_{D}\right)$, and such that the relation which interprets the equality symbol in this new interpretation is denoted by $\approx_{D^{\prime}}$, let $f: D \longrightarrow D^{\prime}$ be the canonic mapping, defined as follows, which associates to every $x \in D$ its equivalence class $f(x) \in D^{\prime}$ (that is, the equivalence class $f(x)$ to which $x$ belongs); thus, $f$ satisfies:

(i) $f(x) \approx_{D^{\prime}} f(y)$ if and only if $x \approx_{D} y$

(ii) For every $n$-ary predicate letter $R$ of a first order language, if $\rho(R)=R^{D}$ and $\rho^{\prime}(R)=R^{D^{\prime}}$, then $R^{D^{\prime}}\left(f\left(x_{1}\right), \ldots, f\left(x_{n}\right)\right)$ if and only if $R^{D}\left(x_{1}, \ldots, x_{n}\right)$

(iii) For every $n$-ary functional symbol $g$ of the language, if $\rho(g)=g^{D}$ and $\rho^{\prime}(g)=g^{D^{\prime}}$, then $g^{D^{\prime}}\left(f\left(x_{1}\right), \ldots, f\left(x_{n}\right)\right) \quad \approx_{D^{\prime}} f\left(g^{D}\left(x_{1}, \ldots, x_{n}\right)\right)$

(iv) For every individual constant $c$, we have that $c^{D^{\prime}} \approx_{D^{\prime}} f\left(c^{D}\right)$

Then, we can prove that the structures $\mathcal{A}$ and $\mathcal{A}^{\prime}$ are elementarily equivalent, that is, every sentence $\alpha$ of the first order language $L$ is such that (in standard notation):

$$
\mathcal{A} \models \alpha \text { iff } \mathcal{A}^{\prime} \models \alpha
$$

The details may be found in Hodges (1983, pp. 68ff), but what the elementary equivalence between the structures says is that that what holds for $x_{1}, \ldots, x_{n}$ in $\mathcal{A}$, holds also for $f\left(x_{1}\right), \ldots, f\left(x_{n}\right)$ in $\mathcal{A}^{\prime}$. As also shown in Mendelson $(1997$, p. 100), the existence of such an $f$ depends essentially of the validity of F2. 
This intuitively says that every element of the domain of $\mathcal{A}^{\prime}$ (which is an equivalence class, hence, a collection of elements of the domain) acts as an individual of the domain of $\mathcal{A}$. So, from the point of view of the language $L$, it is not possible to know whether we are considering an element of $D$ or an equivalence class in $D^{\prime}$ (a certain collection of elements of $D$ ). For uniquely characterizing the diagonal $\Delta_{D}$, we need second order variables, that is, variables which run over collections of objects of $D$ or, alternatively (in extensional contexts), over their properties. But even in this case there are details to be paid attention to, as we shall remark below, for the use of second-order variables is not enough; we need to consider also the meta-mathematical framework where the play is being performed. ${ }^{4}$

Another way of seeing the limitations of first order languages for characterizing identity is the following one. Suppose we have Leibniz Law (the conjunction of (1) and (2) above) in mind and try to capture it as close as we can in a first order language. ${ }^{5}$ So, we should be tempted to write something like the following schema, since we cannot quantify over predicates: for every $F$ denoting a predicate of individuals, we have:

$$
a=b \leftrightarrow(F(a) \leftrightarrow F(b)) .
$$

But suppose now that we have a domain $D$ with cardinality $\aleph_{0}$ and that our first order language has a countable number of monadic predicates, so as that $a$ and $b$ name two elements of $D$, which we denote by $\rho(a)$ and $\rho(b)$, according to the above notation. But, as implied by the axioms of the underlying set theory (suppose Zermelo-Fraenkel), which is the ambient theory where the interpretation is supposed to be defined, $\rho(a)=\rho(b)$ if and only if for every subset $X$ of the domain, $\rho(a) \in X$ if and only if $\rho(b) \in X$. Since in standard semantics $F(a)$ is true if and only if $\rho(a) \in F^{D}$ (the extension of $F$ ), then the right side of the schema (7) refers to at most $\aleph_{0}$ subsets of $D$, while we know that $D$ (with such a cardinality) has $2^{\aleph_{0}}$ subsets. Hence, even if the right side of (7) holds, this fact does not ensure that $\rho(a)$ and $\rho(b)$ are the very same element of $D$, for necessarily there is a subset $Y \subseteq D$ which is not the extension of any predicate of the language under the given interpretation, and such that $\rho(a) \in Y$ but $\rho(b) \notin Y$. This is essentially why there may exist models for a second order language where Leibniz Law is true but where $a$ and $b$ (consider again the conjunction of (1) and (2)) have not the same referent, as remarked above. Denumerable first order languages have limitations also from this point of view.

In regarding first order languages, we still remark that they are also not generally adequate for studying in full the relationships between objects of a certain domain, except if this domain is finite, which is not the case with the

\footnotetext{
${ }^{4}$ Of course, to mention in brief, in the semantics for higher-order logics (Henkin style) we may find models for a language so that even Leibniz Law being true (equation 8 below), $a$ and $b$ may be not 'the very same object'; see (Robin 1969, p. 144, exercise 1).

${ }^{5}$ That is, we may be trying to write down the 'metaphysical' conception of identity mentioned at note 4 .
} 
most interesting mathematical theories, which are usually committed to mathematical constructs like real numbers and the like, that is, with infinite domains. Indeed, it follows from the Löwenheim-Skolem theorems that if a structure has an infinite domain, then it cannot be characterized by a first order theory (the particular case involving identity was mentioned above). Of course LöwenheimSkolem theorems do not hold in higher order logics, but other kinds of problems arise in such contexts, as we shall mention below.

\section{The case of higher-order languages}

In higher order logics the definition of identity usually has motivation in Whitehead and Russell's definition given in Principia Mathematica (1927, p. 57). Inspired by their work, the definition may be written as follows:

$$
a=b={ }_{\mathrm{df}} \forall F(F(a) \rightarrow F(b)) .
$$

We remark that in the definiens that what appears is the material implication, and not a biconditional, as in the earlier mentioned Leibniz Law. Intuitively speaking, as remarked by Boolos and Jeffrey (1989, p. 200, where the formal details are also given), the motive is that (8) "is valid because among the properties of $a$ is the property of being identical with $a$ [which can be defined as $\left.I_{a}(x) \leftrightarrow x=a\right]$; then, if $b$ is to have all of $a$ 's properties, it must have that one in particular". This kind of remark could be taken as quite obvious by someone, but sometimes the property 'being identical with $a$ ' is put in doubt as a legitimate (relational) attribute of $a$ in the context of the different forms of PII; cf. section 1 above; see (French 1999). Then, in considering these situations, we should pay attention to the way we have stated the definition of identity; without this 'problematic' property (namely, the property of 'being identical with $a^{\prime}$ ) in the range of $X$, definition (8) needs the biconditional. This remark will have consequences in what follows, mainly in regarding the situation when dealing with non-rigid structures, as we shall see below.

\section{A way of characterizing indiscernibility}

Cantor's informal characterization of the concept of set states that "by an aggregate we are to understand any collection into a whole of definite and separate objects of our intuition or of our thought" (Cantor 1955, p. 85). ${ }^{6}$ The axiomatic

\footnotetext{
${ }^{6}$ It is worth noting that Cantor's original 'definition' of set at page 204 of his Gesammelte Abhandlungen, of which the above definition is a restatement, does not display such a commitment with individuality tout court, for it reads: "a set is a many which can be thought as a one". So, at least in principle, we might guess that in its very beginnings, set theory does not rule out collections of indiscernible objects, for nothing in Cantor's quotation in this footnote entails that the elements of a set should be 'individuals', as the subsequent ways of expressing the idea of set (and of course the axiomatic versions) did -as that one mentioned in the text.
} 
versions of set theory (Zermelo-Fraenkel, von Neumann-Bernays-Gödel etc), despite their differences, in certain sense 'accept' this characterization, and hence classical mathematics do it too. The important point to be emphasized here is that for the Axiom of Extensionality to hold, it is necessary to have a criterion for two elements being the same object. In other words, set theories display a theory of identity for both the elements of a set and for the sets themselves, provided that it makes any difference (usually given by the underlying first order logic plus the Axiom of Extensionality).

As we shall make explicit below, if the theory admits the existence of entities which are not sets, but that may be elements of a set, that is, the Urelemente, then we can consider them as indistinguishable, as Fraenkel did, but this is to be taken in the sense that whatever permutation of Urelemente can be extended to an automorphism of the universe (this example will be recalled again below). What we would like to remark is that even in this case the Urelemente are subjected to the theory of identity imposed by the underlying logic, and hence they cannot be taken as genuine indiscernible objects, differing solo numero. Independently whether set theory is treated as a first order theory, as it has been usual ever since Skolem, or is axiomatized having (say) a second order logic in its bases, the involved theory of identity is essentially that one sketched in the previous sections. So, being $a$ and $b$ either Urelemente or sets, it makes sense to assert that either $a=b$ or $a \neq b$ holds. In the last case, there exists a set (which in extensional contexts can be associated to a 'property') that 'separates' $a$ from $b$, that is, a set $X$ such that $a$ belongs to $X$ iff $b$ does not. Hence, the intuitive concept of individuality makes sense to all objects of the domain, independently of whether or not we can actually realize the distinction between them.

Classical logic and mathematics pass away of the consideration of ab ovo indistinguishable objects in a way that Cantor's original definition mentioned at footnote 6 arguably does not. In short, the standard ways of considering things as indiscernible starts with individuals of a kind, for instance, with elements of a set (here, by a set we understand a mathematical entity 'given' by the axioms of a set theory like Zermelo-Fraenkel) and then postulate some conditions or operations on these objects so that they look as indiscernible; the necessity of these moves are, in our opinion, due to the assumptions of the underlying mathematical language (including logic), which is in essence a 'language of individuals', as we have said. This means that within the context of 'standard' mathematics (ZF set theory, say), the most we can do is to find a way of saving the appearences, in the sense of providing a way of pretending that some distinguishable entities can be treated as if they were indistinguishable.

Just to exemplify, let us mention one of such possibilities by mentioning Hermann Weyl's considerations on aggregates of individuals.

\subsubsection{Weyl's strategy}

In considering 'aggregates of individuals' for discussions in the foundations of quantum theory, Hermann Weyl has examined the case where the elements of 
a certain collection may be in certain 'states' but only the quantity of them in each of these states would be known (Weyl 1949, App. B). Weyl's strategy is a nice example of what happens with the standard treatments of indiscernibility. According to him, this is what happens in quantum physics. Weyl has taken a set $S$ (let us emphasize this fact) with $n$ elements, say $x_{p_{1}}, \ldots, x_{p_{n}}$, endowed with an equivalence relation $\sim$. The elements $C_{1}, \ldots, C_{k}$ of the quotient set $S / \sim$ were taken to stand for the 'states', and since each one of them has a cardinal $n_{i}, i=1, \ldots, k$, 'the important thing' to be considered is that there is a certain quantity of elements of $S$ in each 'state', which is achieved by considering the ordered decomposition $n_{1}+\cdots+n_{k}=n$. Then, Weyl has suggested to take for granted this decomposition, and 'to forget' that the involved objects which are being counted are elements of a set, hence 'individuals' of a kind (he didn't use these words, but the consequences are precisely these ones). In doing that, Weyl suggested that we arrive at a situation where

"... no artificial differences between elements are introduced by their labels $p$ and merely the intrinsic differences of state are made use of ...". (Weyl 1949, p. 239)

Of course there is a gap here. Weyl's alternative of considering a set endowed with an equivalence relation does not work in characterizing indistinguishability. This could be done only if by some strategy we were able to begin with the above ordered decomposition, but we can't forget that $S$ was taken as a set right from the start after all, so that its elements are distinguishable entities, at least in principle, as it results from Cantor's second 'definition' referred to above in the text, so as from whatever standard axiomatic set theory we may use (ZF, NBG etc.) for formally describing all of this, as seen above. So, for arriving at Weyl's conclusion we should agree that the (at least in principle) 'identifiable' characteristics of the elements of $S$ were masked by the mathematical trick of abstracting the fact that they are elements of a set, hence individuatable entities, and that what was taken into account was only their role as elements of a certain equivalence class. Of course this may work for mathematical purposes, but from the philosophical point of view it seems to us that something more is in need for characterizing 'legitimate' indiscernibility right from the start.

Anyway, let us explore with more details the mathematical treatment of indiscernible objects.

\section{Indiscernibility in a structure}

In mathematical contexts, it is possible to characterize a notion of indistinguishability also by considering the idea of invariance under automorphisms. But, in doing so, we became committed to indiscernibility relative to a certain structure; let us give some details on this point, which we hope will illuminate some aspects which underly the philosophical discussion on individuation. In this direction, we found Fraenkel, Bar-Hillel and Levy saying that (Fraenkel et al., p. 59) 
"(...) there is no characteristic which distinguishes one individual from another (...) in mathematical terms one would say that every permutation of the individuals can be extended to an automorphism of the universe of elements."

The individuals they refer to are the Urelemente of ZFU (the ZermeloFraenkel with Urelemente set theory). The above quotation refers to the role played by the Urelemente in Fraenkel's proof of the consistency of the negation of the Axiom of Choice with the remaining axioms of ZFU (excluding the Axiom of Foundation). As is well known, in this proof Fraenkel admitted the existence of a denumerable infinite collection of Urelemente (Fraenkel 1922).

The concept of invariance under automorphisms was also noted by others like Krasner and Sebastião e Silva (Krasner 1938; Silva 1944). For instance, the Portuguese mathematician José Sebastião e Silva in 1944 said that

"if an element [of a certain structure] cannot be individualized, and hence logically discernible from other elements (as the number $i$ is indiscernible from $-i$ by means of the usual primitive notions), it seems that there is an automorphism of the system which carries this element in any one of the others." (Sebastião e Silva 1944, p. 281). ${ }^{7}$

A way of understanding how the above intuitive notion of indistinguishability acts is to relate it with another important concept, namely, that one of absolute definability. Let us provide the grounds for seeing that.

The following passage, taken from Rogers (1967), helps in fixing the main idea; as he says,

"[i]s there an absolute notion of definability? Although logicians, in their concern with particular formal systems, have largely ignored it, a natural notion for absolute definability has been current in mathematics for some time. This is the notion of invariance under automorphisms (...) We say that $V \subseteq \mathcal{V}$ [where $\mathcal{V}$ is the domain of a structure] is invariant under all automorphisms if $f(V)=V$ for every automorphism $f$ [of that structure]. It is clear that if $V$ is to be "definable" (in some sense) in a given structure, it must be invariant under all automorphisms of the structure; for $f(V)$ must satisfy any "definition" that $V$ satisfies. Conversely it can be argued that the invariant subsets of $\mathcal{V}$ are just the sets which are determined (in some sense) by the structure, and hence that they should be called 'definable'."

So, let us state a definition. If

$$
\mathcal{A}=\left\langle D,\left\{R_{i}\right\}_{i \in I}\right\rangle
$$

${ }^{7}$ We thank Prof. Newton da Costa for pointing us these references. 
is a structure, let $a$ and $b$ be elements of $D$ (the domain of the structure). Then we say that $a$ and $b$ are $\mathcal{A}$-distinguishable (or distinguishable in the structure $\mathcal{A})$ if and only if there exists a sub-collection $X \subseteq D$ such that:

(i) $X$ is invariant under automorphisms of $\mathcal{A}$, that is, $f(X)=X$ for every automorphism $f$ of $\mathcal{A}$, and

(ii) $a \in X$ if and only if $b \notin X$,

otherwise, we say that $a$ and $b$ are $\mathcal{A}$-indistinguishable. That is, $a$ and $b$ are $\mathcal{A}$ indistinguishable if and only if for every sub-collection $X \subseteq D$, if $X$ is invariant under automorphisms of $\mathcal{A}$, then $a \in X$ if and only if $b \in X$.

In the standard extensional set theoretical tradition, we may (roughly speaking) identify a property of objects with a collection of objects, precisely the collection of the objects which have that property. For instance, the property of being a natural number is identified with the set of natural numbers and the property of being an ordinal is identified with the class of ordinals. In such a framework, we may link the above definition of $\mathcal{A}$-distinguishability with the aforesaid concept of absolute definability; the idea is to say that two elements are $\mathcal{A}$-indistinguishable in a given structure if and only if they share all the absolutely definable properties of this structure, that is (in set theoretical terms), when they belong to the same collections of elements of the domain that are invariant under automorphisms.

As it is easy to see, we can restate the above definition in the following equivalent way: $a$ and $b$ are $\mathcal{A}$-indistinguishable if and only if there exists an automorphism $f$ of the structure $\mathcal{A}$ such that $f(a)=b$. Indeed, if $f(a)=b$ for some automorphism $f$ of $\mathcal{A}$ and if $X \subseteq D$ is invariant under automorphisms of $\mathcal{A}$, then $a \in X$ if and only if $b \in X$, for $f(a)=b$ and $f^{-1}(b)=a$. So, $a$ and $b$ are $\mathcal{A}$-indistinguishable. Conversely, if $f(a)=b$ does not hold for any automorphism of $\mathcal{A}$, then $X=\{g(a): g$ is an automorphism of $\mathcal{A}\}$ is a subcollection of the domain which is invariant under automorphisms of $\mathcal{A}$ such that $a \in X$ and $b \notin X$. So $a$ and $b$ are $\mathcal{A}$-distinguishable.

Of course this kind of indistinguishability is not equivalent to identity in all structures (here, by identity we understand the diagonal of the domain), but it is in agreement with the intuition of the mathematician and clarifies some philosophical aspects involved in this topic. Let us try to clarify a little bit this last idea.

We recall that a structure $\mathcal{A}$ is rigid if and only if its only automorphism is the identity function. It is clear that in a rigid structure, every subset of the domain is invariant under automorphisms. So, given $a$ and $b$ in the domain, with $a \neq b$, then $a$ and $b$ are $\mathcal{A}$-distinguishable, since $\{a\}$ is invariant under automorphisms, $a \in\{a\}$ but $b \notin\{a\}$. Furthermore, if $\mathcal{A}$ is a structure where $\mathcal{A}$-indistinguishability and identity coincide, that is, $a$ and $b$ are $\mathcal{A}$-indistinguishable if and only if $a=b$, then $\mathcal{A}$ is rigid. The proof is easy to state. Suppose that $f$ is an automorphism of $\mathcal{A}$ which is not the identity function. Then there exists an $a$ in the domain such that $f(a)=b \neq a$. But, since $b \neq a$ and in such a structure by hypothesis identity and $\mathcal{A}$-indistinguishability 
coincide, then $a$ and $b$ are $\mathcal{A}$-distinguishable, that is, there exists a sub-collection $X$ of the domain such that: (i) $X$ is invariant under automorphisms; (ii) $a \in X$ but $b \notin X$. But this is a contradiction, for being $X$ invariant under automorphisms and $a \in X$, we should have $f(a)=b \in X$.

So, rigid structures are precisely those structures in which we may use the property "to be identical with $a$ " for characterizing $a$ and for distinguishing it (in such structures) from the other objects of the domain, by using the just mentioned concept of distinguishability in a structure. Let us give some examples.

(i) If $\mathcal{A}=\langle A,<\rangle$ is a well ordered structure then, as it is well known, $\mathcal{A}$ is rigid. Really, if $f$ is an automorphism of $\mathcal{A}$ which is not the identity function, then there exists a least element $a \in A$ such that $f(a) \neq a$. Of course, we can't have $f(a)=x<a$, since for every $x<a$, we have $f(x)=x$. Then $f(a)>a$ but, since $f$ is surjective, there exists a $b \in A$ such that $f(b)=a$. But neither $b=a$, for $f(a) \neq a$, nor $b<a$, for $f(x)=x$ for every $x<a$. Hence $b>a$ and $f(a)>f(b)$, which contradicts $f$ being an automorphism.

This shows that every ordinal is a rigid structure. Frequently, when we are dealing with a certain collection of $n$ (in principle) indistinguishable objects and we need to make them distinguishable by some motive, as for instance for talking of them, ${ }^{8}$ generally what we do is to associate an ordinal to the collection of these objects, which corresponds to say something like "let $o_{0}, o_{1}, o_{2}, \ldots, o_{n-1}$ be such objects". For instance, in considering two 'identical' (in the physicist's jargon) electrons, then in order to write down the wave function for the joint system we usually label them with 'names', say particle \#1 and particle \#2 (Teller 1995, pp. 21ff). The notion of indistinguishability in a structure, at least in principle, makes clear what we are doing when we label the particles: we associate the considered objects with the elements of the domain of a rigid structure. But now we note that this is a kind of reverse of the famous (or infamous) process of abstraction. In such a process, we progressively ignore distinctions. Here, we introduce distinctions by means of labelling. This point will be discussed again at the end of the paper.

(ii) Let $\mathcal{A}=\langle Z,+\rangle$ the additive group of the integers. Then $\mathcal{A}$ is, of course, not rigid, for $f: \mathbf{Z} \rightarrow \mathbf{Z}$ defined by $f(x)=-x$, for every $x \in \mathbf{Z}$, is an automorphism, the only one which is not the identity function. So, for every $x \in \mathrm{Z}, x$ and $-x$ are $\mathcal{A}$-indistinguishable.

(iii) Let $V$ be a Zermelo-Fraenkel (ZF) universe. Then, by the isomorphism theorem (Jech 1997, p. 74 ), $\mathcal{A}=\langle V, \in\rangle$ is rigid. ${ }^{9}$ This agrees with the idea that in the standard mathematics (that is, that one built in ZF), identity

\footnotetext{
${ }^{8}$ This relationship with the needs of language was emphasized for instance by G. Toraldo di Francia, who said that 'objectuation' is a primitive act of understanding. According to him, we divide up the world in 'objects' (individuals) to speak of them; see Toraldo di Francia 1981, p. 222 .

${ }^{9}$ We recall that if $V$ is the usual well-founded universe of $\mathrm{ZF}$ (the class of all well-founded sets), then of course $V$ is not a set but still so we can talk of automorphisms of $\langle V, \in\rangle$.
} 
and indistinguishability coincide, as we have said above, for identity and $\mathcal{A}$ indistinguishability coincide.

(iv) Let $U$ be a Zermelo-Fraenkel with Urelemente (ZFU) universe. Then, $\mathcal{A}=\langle U, \in\rangle$ is not rigid. ${ }^{10}$ Really, whatever permutation of the Urelemente can be extended to an automorphism of $\mathcal{A}$ (Jech 1997, pp. 198-199). As we have said above, this fact is in the core of Fraenkel's proof of the consistency of the negation of the Axiom of Choice (with the remaining axioms of ZFU, except Foundation), and in a certain sense justifies the standard view that the Urelemente, despite not identical, are indistinguishable. ${ }^{11}$

Furthermore, there is an analogy between the presentation of Urelemente as distinct objects and the remarks made above (at example (i)) concerning the introduction of distinctions between two particles by the use of labels. Paul Cohen, in his book on set theory presents the Urelemente this way:

"We next discuss models $N$ in which AC [the Axiom of Choice] fails. Classical results in this direction were obtained by Fraenkel and Mostowski, who dropped the Axiom of Extensionality and introduced 'atoms' [that is, Urelemente], i.e., fictitious objects $x_{i}$ such that $\forall y\left(\neg y \in x_{i}\right)$ yet $x_{i} \neq x_{j}$ for $i \neq j . "$ (Cohen 1966, p. 136)

So, Urelemente are distinguished by their labels. If we try to characterize the differences between Urelemente $a$ and $b$ sayng that $a \neq b$ if and only if $a$ and $b$ don't belong to the same sets, we fall in a kind of circularity. For instance, $a \in\{a\}$, but in order to know whether or not $b$ belongs to $\{a\}$, we must know in advance whether or not $b$ is equal to $a$. It is interesting to note that the same kind of circularity was noted by E. J. Lowe, who, concerning D. Davidson's criterion of identity of events, wrote:

"But there certainly can be such circularity, as for instance in Donald Davidson's one-level criterion of identity for events (...): ${ }^{12}$

$$
\begin{gathered}
\forall x \forall y(E(x) \& E(y) \rightarrow(x=y \leftrightarrow \forall z(E(z) \rightarrow \\
(C(x, z) \leftrightarrow C(y, z)) \&(C(z, x) \leftrightarrow C(z, y)))))
\end{gathered}
$$

In words: if $x$ and $y$ are events, then $x$ is identified with $y$ if and only if $x$ and $y$ cause and are caused by the same events, This is circular inasmuch as what makes for sameness amongst events is precisely what a criterion of identity for events is supposed to convey, and yet

\footnotetext{
${ }^{10}$ Similar remarks made for the previous case are in order here.

${ }^{11}$ If pressed, a logician could say that the Urelemente are members of a set of sets of the same rank in an adequate model of the Zermelo-Fraenkel set theory (Jech 1977, p. 199). This convenient option of using a theory without indistinguishable objects to explain the status of indistinguishable objects occurring in another theory is not, in our opinion, generally open to the physicist regarding elementary particles. It is not easy to see how to explain their indistinguishability in terms of, for instance, distinguishable classical objects.

${ }^{12}$ We have simplified the notation, writing $C(x)$ for Cause $(x)$ and $E(x)$ for Event $(x)$.
} 
a grasp of that is needed in order to understand what is expressed on the right-hand side of the main biconditional [in the above sentence]. (Lowe 1999)

As usual, Lowe doesn't see the same problem with Urelemente and, in fact, there is no problem with them provided we accept distinctions by means of labelling as legitimate ones.

Let us consider now the following definitions. If $\mathcal{A}=\left\langle D,\left\{R_{i}\right\}_{i \in I}\right\rangle$ is a structure, we say that a structure $\mathcal{B}$ is an expansion of $\mathcal{A}$ if and only if $\mathcal{B}=$ $\left\langle D,\left\{R_{i}\right\}_{i \in I \cup J}\right\rangle$, where $I \cap J=\emptyset$. In other words, $\mathcal{B}$ is an expansion of $\mathcal{A}$ if and only if $\mathcal{B}$ is obtained by adding new relations to $\mathcal{A}$. For example, $\langle Z,+,<\rangle$ is an expansion of $\langle Z,+\rangle$. We say that $\mathcal{B}$ is a trivial rigid expansion of $\mathcal{A}$ if and only if the following clauses are met: (i) $\mathcal{B}$ is an expansion of $\mathcal{A}$; (ii) $\mathcal{B}^{\prime}=\left\langle D,\left\{R_{i}\right\}_{i \in J}\right\rangle$ is rigid (in this case, $\mathcal{B}$ is also rigid). This means that the new relations added to $\mathcal{A}$ in order to obtain $\mathcal{B}$ are, alone, sufficient to ensure the rigidity of $\mathcal{B}$ regardless of the original relations of $\mathcal{A}$. For example, if $\mathcal{A}=\langle Z,+\rangle$, then $\mathcal{B}=\langle Z,+,\{0\},\{1\},\{-1\}, \ldots\rangle$ is a trivial rigid expansion of $\mathcal{A}$, since $\mathcal{B}^{\prime}=\langle Z,\{0\},\{1\},\{-1\}, \ldots\rangle$ is obviously rigid.

It is easy to see that every structure has a trivial rigid expansion, for instance, that one obtained by adding to it all the singletons of the elements of its domain, as we have done above with $\langle Z,+\rangle$. Let us also remark that given a structure $\mathcal{A}=\left\langle D,\left\{R_{i}\right\}_{i \in I}\right\rangle$, another way of obtaining a trivial rigid expansion of $\mathcal{A}$ is to take $\mathcal{B}=\left\langle D,\left\{R_{i}\right\}_{i \in I},<\right\rangle$, where $<$ is a well-ordering of $D$ (the existence of such a well-ordering is granted by the Axiom of Choice, or by the Axiom of Global Choice).

Finally, we say that a structure $\mathcal{B}$ is a non-trivial rigid expansion of $\mathcal{A}=$ $\left\langle D,\left\{R_{i}\right\}_{i \in I}\right\rangle$ if and only if (i) $\mathcal{B}$ is an expansion of $\mathcal{A}$, (ii) $\mathcal{B}$ is rigid, but (iii) $\mathcal{B}^{\prime}=\left\langle D,\left\{R_{i}\right\}_{i \in J}\right\rangle$ is not rigid. In this case, the new relations added to $\mathcal{A}$ are not, alone, sufficient to ensure the rigidity of $\mathcal{B}$ regardless the original relations of $\mathcal{A}$. For example, if $\mathcal{A}=\langle\mathrm{Z},+\rangle$, then $\mathcal{B}=\langle\mathrm{Z},+,<\rangle$ is a non-trivial rigid expansion of $\mathcal{A}$, since $\langle\mathbf{Z},+,<\rangle$ is rigid because the only automorphism of $\langle\mathbf{Z},+\rangle$ distinct from the identity function is $f: Z \mapsto Z$ defined by $f(x)=-x$, which does not preserve the order $<$. But $\langle\mathbf{Z},<\rangle$ is not rigid, since, for each $k \in \mathbf{Z}$, the function $f_{k}: \mathrm{Z} \mapsto \mathrm{Z}$ defined by $f_{k}(x)=x+k$ is an automorphism of $\langle\mathrm{Z},<\rangle$. As another example, take again $\mathcal{A}=\langle\mathbf{Z},+\rangle$. Then $\mathcal{B}=\langle\mathbf{Z},+,\{k\}\rangle$, where $k \in \mathbf{Z}$ is a fixed but arbitrary non-zero element of $Z$, is a non-trivial rigid expansion of $\mathcal{A}$, for although $\mathcal{B}$ is rigid (again, the only automorphism of $\langle Z,+\rangle$ distinct from the identity function does not preserve $\{k\}$ for $0 \neq k \in \mathbf{Z}), \mathcal{B}^{\prime}=\langle Z,\{k\}\rangle$ is not, since any permutation of $Z$ that fixes $k$ is an automorphism of $\langle Z,\{k\}\rangle$. On the other hand, $\langle Z,+,\{0\}\rangle$ is not a non-trivial rigid expansion of $\langle Z,+\rangle$, because it is not rigid. An example of a structure that doesn't have a non trivial rigid expansion is $\mathcal{A}=\langle D, R\rangle$, where $D=\{1,2\}$ and $R=\{\langle 1,2\rangle,\langle 2,1\rangle\}$. Indeed, $f: D \mapsto D$ defined by $f(1)=2$ and $f(2)=1$ is an automorphism of $\mathcal{A}$, so this structure is not rigid. If $\mathcal{B}$ is a rigid expansion of $\mathcal{A}$, then the relations added to $\mathcal{A}$ in order to form $\mathcal{B}$ must, alone, render $f$ into a non-automorphism, but in doing this, these relations are, alone, sufficient to ensure the rigidity of $\mathcal{B}$, since 
the only permutation of $D$, other than $f$, is the identity function.

These definitions will enable us to consider some philosophical points from a 'formal' perspective.

\section{Physics and metaphysical alternatives}

The development of quantum statistics has brought the claim that quantum particles cannot be regarded as on a par with 'macroscopic' objects like rocks and people. According to some scholars, they are, in some sense, 'non-individuals' (Schrödinger 1950/1998; Weyl 1949). A way of making sense to this idea is to consider that the concept of identity does not make sense to them, as explicitly emphasized by Schrödinger (1952, pp. 17-18). But an alternative view has also been defended, by saying that, on the contrary, quantum particles can, in fact, be regarded as individuals, albeit with very different properties and behavior from the classical ones. As put by S. French, this gives rise to a very interesting situation (not explored here) according to which our fundamental metaphysics is underdetermined by the physics (French 1999). In considering these views, we can distinguish between two main lines of thought in what concerns indistinguishable particles and analyse them from the perspective of what we have said in the previous section. So, let us sum up them, very briefly, as follows:

(A) The first hypothesis says that elementary particles of the same kind are indistinguishable in an ontological sense. E. J. Lowe, among others, sustains such a view, which has been discussed in several papers which have appeared in the last years, mainly in Analysis. ${ }^{13}$ According to this view, particles in a superposition state cannot be distinguished, even in principle. This fits well the idea of non-individuals, put by several authors like those mentioned above, and apparently is in accordance with the basic suppositions of quantum field theory (Krause and French 1995).

(B) According to the second hypothesis, elementary particles of the same kind are indistinguishable from an epistemological point of view only. This idea may be related to some kind of hidden variables theory, for we may suppose that the progress of physics, logic etc., will tell us what should be the relevant properties that serve to distinguish among these particles (Sant'Anna 2000). This view may also be merged with some aspects of van Fraassen's constructive empiricism, despite van Fraassen does not endorse hidden variables. Even without discussing van Fraassen's position, we might recall that, according to him, despite 'identical' elementary particles are indistinguishable from the point of view of all the means provided by quantum theory, even so they can be distinguished: "[identical particles in the same state of motion] are certainly qualitatively the same in all respects representable in quantum mechanical models

\footnotetext{
${ }^{13}$ For instance, see Lowe 1994, Noonan 1995, Odrowaz-Sypniewska 2001, to mention few of them. See also French and Krause 2002.
} 
-yet still numerically distinct" (van Fraassen 1991, p. 376). This strange possibility is related to the ways out van Fraassen offers to this dilemma: either the principle of the identity of indiscernibles is violated or quantum mechanics is not complete. As is well known, both possibilities raise a cluster of philosophical problems. van Fraassen maintains PII, since he distinguishes between quantum dynamical states and experimental events; the former are that what a vector or a statistical operator represents, and are embedded in the theory, whose evolution is governed by dynamical laws. In other words, dynamical states are described within the formalism of quantum mechanics. Experimental events, on the contrary, are extra-theoretical entities which respect the ways the probabilities calculations are performed. The same conceptual distinction is made by distinguishing between state attributions and value attributions of a physical system. The former, roughly, is a theoretical construct, and part of the problems involved in theory construction depends upon a proper representation of the states, while the latter would be something 'meta-theoretical'. This distinction seems to be interesting and, we guess, may raise further important philosophical insights, for it seems that we should regard events as something 'outside' the theory, so that the distinctness properties of the particles could be achieved only in the meta-theoretical realm. So, PII could be saved in considering the role played by the metalanguage of quantum physics. The intuitions here suggest the consideration, in empirical sciences, of a topic which resembles Skolem's relativism, where a certain set theoretical concept can have different meanings in different models of set theory. In other words, maybe something like the set-theoretical issue of absoluteness could be pursued also in connection with the empirical sciences, but of course the details of such an idea need to be further explored.

In this paper, we will not enter into the detailed philosophical dispute between positions (A) and (B) above but, instead, we will only prepare the grounds for a formal analysis of such a dispute (which we intent to pursue in a future paper) by using the concepts we have presented in the previous section. So, we suggest a model-theoretical characterization of positions (A) and (B), as follows.

Firstly, position (A) amounts to sustain that a mathematical structure of quantum theory ${ }^{14}$ cannot have a non-trivial rigid expansion, which intuitively means that the rigidity of a structure for quantum theory can be achieved only by considering new relations which, by themselves, regardless the quantum nature of the elements of the domain, guarantee such a rigidity. Earlier, we have mentioned labelling as a mean of distinguishing two particles. Such labels play the same role when used to distinguish between two oranges. The nature, classical or quantum, of the considered objects does not matter from the point of view of using labels. The only relevant point is that the labels provide a way of associating a well-ordered structure, that is, an ordinal, to the collection of the considered objects.

Position (B), on the contrary, can be considered as a view according to which a mathematical structure of quantum theory has a non-trivial rigid expansion.

\footnotetext{
${ }^{14}$ Here, we are, of course, endorsing in the fiction that there is such an structure.
} 
That is, in this case we are accepting that the objects of the domain (among of them, the elementary particles) may be distinguished one from the others, but not merely by posing new relations such as well-orders, i.e., labels, that by themselves are alone responsible for such distinguishability (discernibility).

We can motivate these views by considering the following passage, in which Schrödinger gives an intuitive account of Maxwell-Boltzmann and Bose-Einstein statistics:

"Three school boys, Tom, Dick, and Harry, deserve a reward. The teacher has two rewards to distribute among them. Before doing so, he wishes to realize for himself how many different distributions are at all possible. (...) It is a statistical question: (...) different kinds of reward will illustrate the (...) kinds of statistics.

(a) The two rewards are two memorial coins with portraits of Newton and Shakespeare respectively. The teacher may give Newton either to Tom or to Dick or to Harry and Shakespeare either to Tom or to Dick or to Harry. Thus there are three times three, that is nine, different distributions (classical statistics).

(b) The two rewards are two shilling-pieces (which, for our purpose, we must regard as indivisible quantities). They can be given to two different boys, the third going without. In addition to these three possibilities there are three more: either Tom or Dick or Harry receives two shillings. Thus there are six different distributions (BoseEinstein statistics).

(...)the rewards represent the particles (...) Memorial coins are individuals distinguished from one another. Shillings, for all intends of purposes, are not, but they are still capable of being owned in the plural. It makes a difference whether you have one shilling, or two (...). There is no point in two boys exchanging their shillings." (Schrödinger 1950/1998)

The idea is that if we build a structure representing this situation, where the property of being a reward is one of its constitutive relations, then there would be no automorphism of this structure carrying one memorial coin to another. But, since, as rewards, the shillings are indistinguishable, there would be an automorphism carrying one shilling to another. This would be the meaning of the last sentence of the above quotation. On the other side, although the shillings, as material objects, can be distinguished from one another, this kind of distinguishability suffices, alone, to identify each shilling. It doesn't involve the property of being a reward. So, a rigid expansion of a structure representing Schrödinger's example, having among its constitutive relations the property of being a material object, is a trivial one, and this doesn't bother those who sustain position (A) above. A successful refutation of position (A), in our opinion, would require a non trivial expansion of a structure representing the quoted example, that is, the consideration of new relations that not alone, but combined with the property of being a reward, produce the rigidity that will allow 
the identification of each shilling (remember the given example of $\langle Z,+\rangle$ and $\langle Z,+,<\rangle)$. It should be remarked that, recently, Nick Huggett has extended Schrödinger's example to more general 'Schrödinger games', which might offer still further possibilities of interpreting positions (A) and (B), but we shall leave this analysis to be discussed in future works (Huggett, forthcoming).

To sum up, we may say that within the context of position (A), the metaphysical view according to which elementary particles are non-individuals can be sustained only in those structures that don't have non-trivial rigid expansions. This informally means that there are theoretical commitments that, in some way, prevent the use of properties like 'being identical with $a$ ' to distinguish $a$ from other objects under consideration. So, what the above discussion perhaps has contributed to show is that the philosophical discussion on the logical foundations of physics must consider the power and the limitations of the underlying logical and mathematical apparatus used in this discussion. Sometimes philosophical problems cannot be rightly analysed only at the informal level, as the standard philosophical discussions usually do. A 'more mathematical' consideration may illuminate some of the problems, as the case of the concepts of trivial and of non trivial rigid expansions of a structure do, according to the above guidelines.

Acknowledgments: We would like to thank Prof. Newton da Costa and the two anonymous referees for useful remarks.

\section{References}

[1] Boolos, G. S. and Jeffrey, R. C.; 1989, Computability and Logic, Cambridge Un. Press, 3rd. ed.

[2] Bueno, O. and French, S.: 1999, 'Infestation or pest control: the introduction of group theory into quantum mechanics', Manuscrito 22 (2), pp. 3768 .

[3] Cantor, G.: 1955, Contributions to the founding of the theory of transfinite numbers, Dover.

[4] Castellani, E.: 1998, 'Galilean particles: an example of constitution of objects', in Castellani, E. (ed.), Interpreting bodies: classical and quantum objects in modern physics, Princeton Un. Press, pp. 181-194.

[5] Church, A.: 1956, Introduction to mathematical logic, Vol. 1, Princeton Un. Press.

[6] Cohen, P. J.: 1966, Set Theory and the Continuum Hypothesis, W. Benjamin.

[7] Fraenkel, A. A.: 1922, 'The notion of 'definite' and the independence of the axiom of choice', reprinted in van Heijenoort, From Frege to Gödel: 
a source book in mathematical logic, 1879-1931, Harvard Un. Press, 1967, pp. 284-289.

[8] Fraenkel, A. A., Bar-Hillel, Y. and Levy, A.: 1973, Foundations of Set Theory, North-Holland.

[9] Frege, G.: 1879, Begriffsschrift, eine der aritmetischen nachgebildete Formalsprache des reinen Denkens, Halle; translated in van Heijenoort, J., From Frege to Gödel: a source book in mathematical logic, 1879-1931, Harvard Un. Press, 1967, pp. 1-82.

[10] Frege, G.: 1884, Die Grundlagen der Arithmetik-Eine logisch mathematische Untersuchung über den Begriff der Zahl, Breslau.

[11] Frege, G.: 1893, Grundgesetze der Arithmetik, begriffsschriftlich abgeleitet, Vol. 1, Jena.

[12] French, S.: 1989, 'Why the principle of the identity of indiscernibles is not contingently true either', Synthese 78, pp. 141-166.

[13] French, S.: 1999, 'Identity and individuality in quantum theory', Stanford Encyclopedia of Philosophy, http://plato.stanford.edu.

[14] French, S. and Krause, D.: 1995, 'Vague identity and quantum nonindividuality', Analysis 55 (1), pp. 20-26.

[15] French, S. and Krause, D.: 2003, 'Quantum vagueness', Erkenntnis 59, pp. 97-124.

[16] Hilbert, D. \& Ackermann, W.: 1950, Principles of Mathematical Logic, Chelsea Pub. Co, NY (reprinted by AMS Chelsea Pu., 1999).

[17] Hodges, W.: 1983, 'Elementary predicate logic', in Gabbay, D. and Guenthner, F. (eds.), Handbook of philosophical logic, D. Reidel, Vol. I, pp. 1-131.

[18] Huggett, N., 'What is an elementary quarticle?', forthcoming.

[19] Jech, T.: 1997, Set theory, Springer, 2nd. ed.

[20] Krasner, M.: 1938, 'Une généralization de la notion de corps', J. de Math. Pures et Appl. 27, pp. 367-385.

[21] Krause, D. and French, S.: 1995, 'A formal framework for quantum nonindividuality', Synthese 102, pp. 195-214.

[22] Lowe, E. J.: 1994, 'Vague identity and quantum indeterminacy', Analysis 54, pp. 101-106.

[23] Lowe, E. J.: 1999, 'Objects and criteria of identity', in Hale, B. and Wright, C. (eds.), A Companion to the Philosophy of Language, Blackwell Pu., pp. 613-633. 
[24] Mendelson, E.: 1997, Introduction to mathematical logic, Chapmann \& Hall, 4th. ed.

[25] Noonan, H. W.: 1995, 'Lowe on vague identity and quantum indeterminacy', Analysis 55, pp. 14-191.

[26] Odrowaz-Sypniewska, J.: 2001, 'Quantum indeterminacy without vague identity', Analysis 61, pp. 65-69.

[27] Robbin, J. W.: 1969, Mathematical logic: a first course, W. A. Benjamin.

[28] Rogers, H.: 1967, 'Some problems of definability in recursive function theory', in Crossley, J. N. (ed.), Sets, models and recursion theory, Proceedings of the Summer School in Mathematical Logic and Tenth Logic Colloquium, Leicester, Aug. - Sept. 1965, North-Holland, pp. 183-201.

[29] Sant'Anna, A. S.: 2000, 'Elementary particles, hidden variables, and hidden predicates', Synthese 125 (1/2), pp. 233-245.

[30] Schrödinger, E.: 1952, Science and humanism, Cambridge Un. Press.

[31] Schrödinger, E.: 1950/1998, 'What is an elementary particle?', reprinted in Castellani, E. (ed.), Interpreting bodies: classical and quantum objects in modern physics, Princeton Un. Press, 1998, pp. 197-210.

[32] Sebastião e Silva, J.: 1944, 'Para uma teoria geral dos homomorfismos', in Obras de José Sebastião e Silva, Lisboa, Instituto Nacional de Investigação Científica, 1985.

[33] Teller, P.: 1995, An interpretive introduction to quantum field theory, Princeton Un. Press.

[34] Toraldo di Francia, G.: 1981, The investigation of the physical world, Cambridge Un. Press.

[35] van Fraassen, B.: 1991, Quantum mechanics: an empiricist view, Clarendon Press.

[36] Weyl, H.: 1949, Philosophy of mathematics and natural science, Princeton Un. Press.

[37] Whitehead, A. N. and Russell, B.: 1927, Principia Mathematica, Cambridge Un. Press, Vol. 1 (2nd. ed. Cambridge Un. Press).

[38] Wigner, E.: 1939, 'On unitary representations of the inhomogeneous Lorentz group', Annals of Mathematics 40, pp. 149-204. 